\title{
Development of a Two-Level Segmentation System for Iris Recognition Using Circular and Linear Hough Transform Algorithm
}

\author{
Olusegun Olayinka Lawal ${ }^{1}$, Babajide Olakunle Afeni ${ }^{2}$, Moshood Olatunji Lawal ${ }^{3}$ \\ ${ }^{1}$ Department of Computer Science, Joseph Ayo Babalola University, Ikeji - Arakeji, Osun State, Nigeria \\ ${ }^{2}$ Department of Computer Science, Joseph Ayo Babalola University, Ikeji - Arakeji, Osun State, Nigeria \\ ${ }^{3}$ Federal Polytechnic, Ede, Osun State, Nigeria
}

\begin{abstract}
Recently, the use of biometric systems is of increasing significance in both public and private sectors. It has gradually replaced traditional system security. Iris recognition biometric systems have proved to be efficient at personal recognition with highest recognition accuracy. In this work a hybrid segmentation technique for iris recognition using circular and linear hough transform algorithms was developed. The hybrid segmentation technique was evaluated in terms of segmentation and recognition time. False Acceptance Rate (FAR) and False Rejection Rate (FRR) were the parameters used for comparison in the iris recognition. The system was implemented in MATLAB. After evaluating the performance of the hybrid segmentation techniques it revealed that the system was faster during the two stages. The averages segmentation time was 0.21089 seconds while average recognition time was 0.04113 seconds. The result shows the approach is efficient for the purpose of authentication.
\end{abstract}

Keywords: System security, authentication, iris, iris recognition, iris segmentation, noise.

\section{Introduction}

With increase in emphasis on security nowadays, biometric technologies are becoming much more important than ever (Peihua and Xiaomin, 2008). In particular, iris recognition in recent years receives growing interests (Adegoke et al.,). Iris recognition is a method of biometric authentication that uses pattern recognition techniques based on high resolution images of the irises of an individual's eye. It uses camera technology, with subtle infrared illumination, to create images of the detail-rich, intricate structures of the iris converted into digital templates; these images provide mathematical representations of the iris that yield unambiguous positive identification of an individual. Iris recognition efficacy is rarely impeded by glasses or contact lenses. Because of its speed of comparison, iris recognition is a biometric technology is well-suited for one-to-many identification. A key advantage of iris recognition is its stability, or template longevity, barring trauma, a single enrollment can last a lifetime. Although small $(11 \mathrm{~mm})$ and sometimes problematic to image, the iris has the great mathematical advantage that its pattern variability among different persons is enormous. In addition, as an internal (yet externally visible) organ of the eye, the iris is well protected from the environment and stable over time. As a planar object, its image is relatively insensitive to angle of illumination, and changes in viewing angle cause only affine transformations; even the non-affine pattern distortion caused by papillary dilation is readily reversible.

The three main stages of an iris recognition system are image preprocessing, feature extraction and template matching. The iris image needs to be preprocessed to obtain useful iris region. Image preprocessing is divided into three steps: iris segmentation, iris normalization, and image enhancement. Iris segmentation detects the inner and outer boundaries of iris. Eyelids and eyelashes that may cover the iris region are detected and removed. Iris normalization converts iris image from Cartesian coordinates to polar coordinates. Feature extraction uses texture analysis method to extract features from the normalized iris image. The significant feature of the iris are extracted for accurate identification purpose. Template matching compares the user template with templates from the database using a matching metric.

Iris segmentation is to locate the valid part of the iris for iris biometrics, including finding the pupillary and limbic boundaries of the iris, localizing its upper and lower eyelids if they occlude and detecting and excluding any superimposed occlusions of eyelashes, shadows or reflections (Lim et al., 2001). The uniqueness and significance of segmentation to effectiveness of any iris recognition system cannot be overemphasized. It determines effectiveness of the system (Nakissa and Mohammad, 2008). According to Daugman (1993), the two most recognised iris segmentation approaches are attributed to Daugman and Wildes. Daugman developed integro-differential operator to find circular pupil and limbus boundaries. It can be interpreted as a circular edge detector, which searches, in a smoothed image by Gaussian filter, the parameters of a circular boundary along which the integra derivative is maximal. Wildes proposed a two-stage iris segmentation method: a gradient based intensity image, and next the inner and outer boundaries are detected using Hough transform (Wildes, 1997). It is reported that most failures to match in iris recognition system result from inaccurate segmentation (Ma, et al., 2004). 


\section{International Journal of Science and Research (IJSR) \\ ISSN (Online): 2319-7064}

Index Copernicus Value (2013): 6.14 | Impact Factor (2014): 5.611

The objective of this research work is to develop an object oriented system that can performs hybrid segmentation technique for iris recognition using Circular Hough transform algorithm. The system will them implemented using the algorithms to measure the accuracy of the hybrid segmentation technique. Finally, the performance evaluation of the system will be carried out.

\section{Literature Review}

A biometric system is essentially a pattern-recognition system that recognizes a person based on a feature vector derived from a specific physiological or behavioral characteristics that the person possesses. That feature vector is usually stored in a database after being extracted. A biometric system based on physiological characteristics is more reliable than one based on behavioral characteristics (Prabhakar et al,2003).

The history of iris recognition goes back to mid-19 century when the French physician, Alphonsus Bertillon, studied the use of eye color as an identifier (Boles and Boashash, 1998). However, it was believed that the main idea of using iris patterns for identification, the way we know it today, was first introduced by an eye surgeon, Frank Burch, in 1936. (Daugman, 2003). In 1987, two ophthalmologists, Flom and San Francisco, patented this idea and proposed it to Daugman, a professor at Harvard University, to study the possibilities of developing an iris algorithm. After a few years of scientific experiments, Daugman proposed and developed a high confidence iris recognition system and published the result in 1993 (Daugman, 2003). The proposed system then evolved and achieved a better performance in time by testing and optimizing it with respect to large databases.

The reports have shown that Daugman's system has zero false matching rates based on the test done by organizations such as British Telecomes, US Sandia Labs, UK National Physical Laboratory, the National Biometric Test Center SJSU (Daugman, 2003). The information extracted from an iris is in binary format and stored in only 256 bytes to allow creation of nationwide Iris Code databases. The search engine is based on Boolean exclusive-OR operator (XOR) to allow extremely fast comparisons in the matching process. The overall characteristics of the proposed algorithm offers real-time and high confidence identification in applications such as passenger control in airports, border control and access control in high-security areas. A few years after the publication of the first algorithm by Daugman, other researchers developed new iris recognition algorithms

\subsection{The Human Iris}

The iris is a thin circular diaphragm, which lies between the cornea and the lens of the human eye. A front-on view of the iris is shown in Figure 2.1. The iris is perforated close to its centre by a circular aperture known as the pupil. The function of the iris is to control the amount of light entering through the pupil, and this is done by the sphincter and the dilator muscle, which adjust the size of the pupil. The average diameter of the iris is $12 \mathrm{~mm}$, and the pupil size can vary from $10 \%$ to $80 \%$ of the iris diameter (Daugman,2002). The iris consists of a number of layers; the lowest is the epithelium layer, which contains dense pigmentation cells. The stromal layer lies above the epithelium layer, and contains blood vessels, pigment cells and the two iris muscles. The density of stromal pigmentation determines the colour of the iris. The externally visible surface of the multi-layered iris contains two zones, which often differ in colour (Wolff, 1976). An outer ciliary zone and an inner pupillary zone, and these two zones are divided by the collarette - which appears as a zigzag pattern.

Formation of the iris begins during the third month of embryonic life. The unique pattern on the surface of the iris is formed during the first year of life, and pigmentation of the stroma takes place for the first few years. The only characteristics that is dependent on genetics is the pigmentation of the iris, which determines its colour. Due to the epigenetic nature of the iris pattern, the two eyes of an individual contain completely independent iris pattern, and identical twins possess uncorrelated iris patterns (Wolff, 1976).

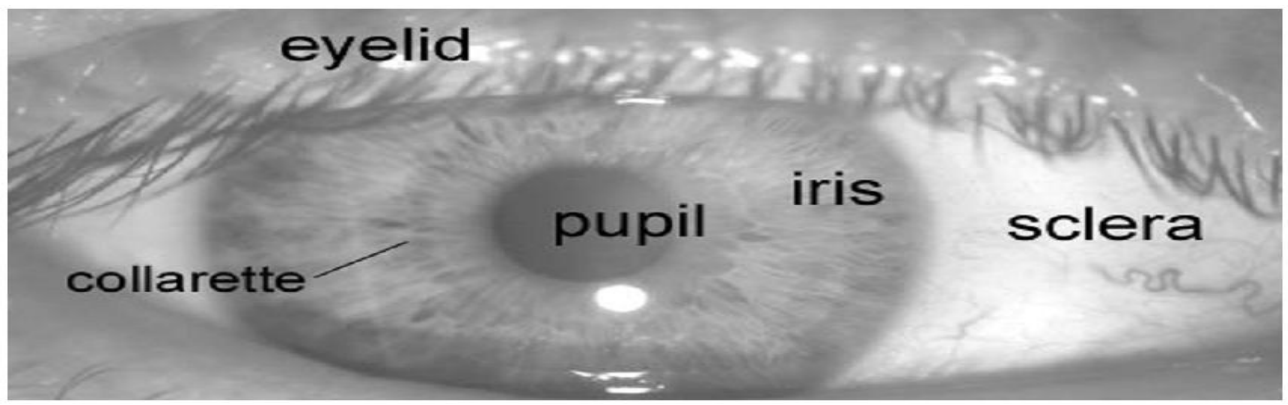

Figure 2.1: A front view of the human eye (Source: Wolff, 1976)

\subsection{Iris Recognition}

The iris is an externally visible, yet protected organ whose unique epigenetic pattern remains stable throughout adult life. These characteristics make it very attractive for use as a biometric for identifying individuals. Image processing techniques can be employed to extract the unique iris pattern from a digitized image of the eye, and encode it into a 


\section{International Journal of Science and Research (IJSR) \\ ISSN (Online): 2319-7064 \\ Index Copernicus Value (2013): 6.14 | Impact Factor (2014): 5.611}

biometric template, which can be stored in a database. This biometric template contains an objective mathematical representation of the unique information stored in the iris, and allows comparisons to be made between templates. When a subject wishes to be identified by iris recognition system, their eye is first photographed, and then a template created for their iris region. This template is then compared with the other templates stored in a database until either a matching template is found and the subject is identified, or no match is found and the subject remains unidentified. In line with the basic steps in Biometric systems operation, iris recognition involves the following processes: Image capturing, Iris Segmentation, Iris Normalization, Feature encoding, and Matching.

\subsection{Image capturing}

The first thing involved is capturing the image of the eye to be used. In capturing, the actual iris pattern, an iris camera is used and it should be at resolution of 70 pixels (Daugman 2003). Special cameras with an illumination of about $70 \mathrm{~mm}-90 \mathrm{~mm}$ in wavelength can also be used. There are also some other techniques used which involves using visual feedback through a mirror or video image to enhance cooperation in that user's position; their eyes within the field of view of cameras.

\subsection{Iris Segmentation}

The first stage of iris recognition is to isolate the actual iris region in a digital eye image. The eyelids and eyelashes normally include the upper and lower parts of the iris region. Also, specular reflections can occur within the iris region corrupting the iris pattern. A technique is required to isolate and exclude these artefacts as well as locating the circular iris region. The success of segmentation depends on the imaging quality of eye images.

\subsection{Iris Normalization}

Once the iris region is successfully segmented from an eye image, the next stage is to transform the iris region so that it has fixed dimensions in order to allow comparisons. The dimensional inconsistencies between eye images are mainly due to the stretching of the iris caused by pupil dilation from varying levels of illumination. Other sources of inconsistency include, varying imaging distance, rotation of the camera, head tilt, and rotation of the eye within the eye socket. The normalization process will produce iris regions, which have the same constant dimensions, so that two photographs of the same iris under different conditions will have characteristic features at the same spatial location.
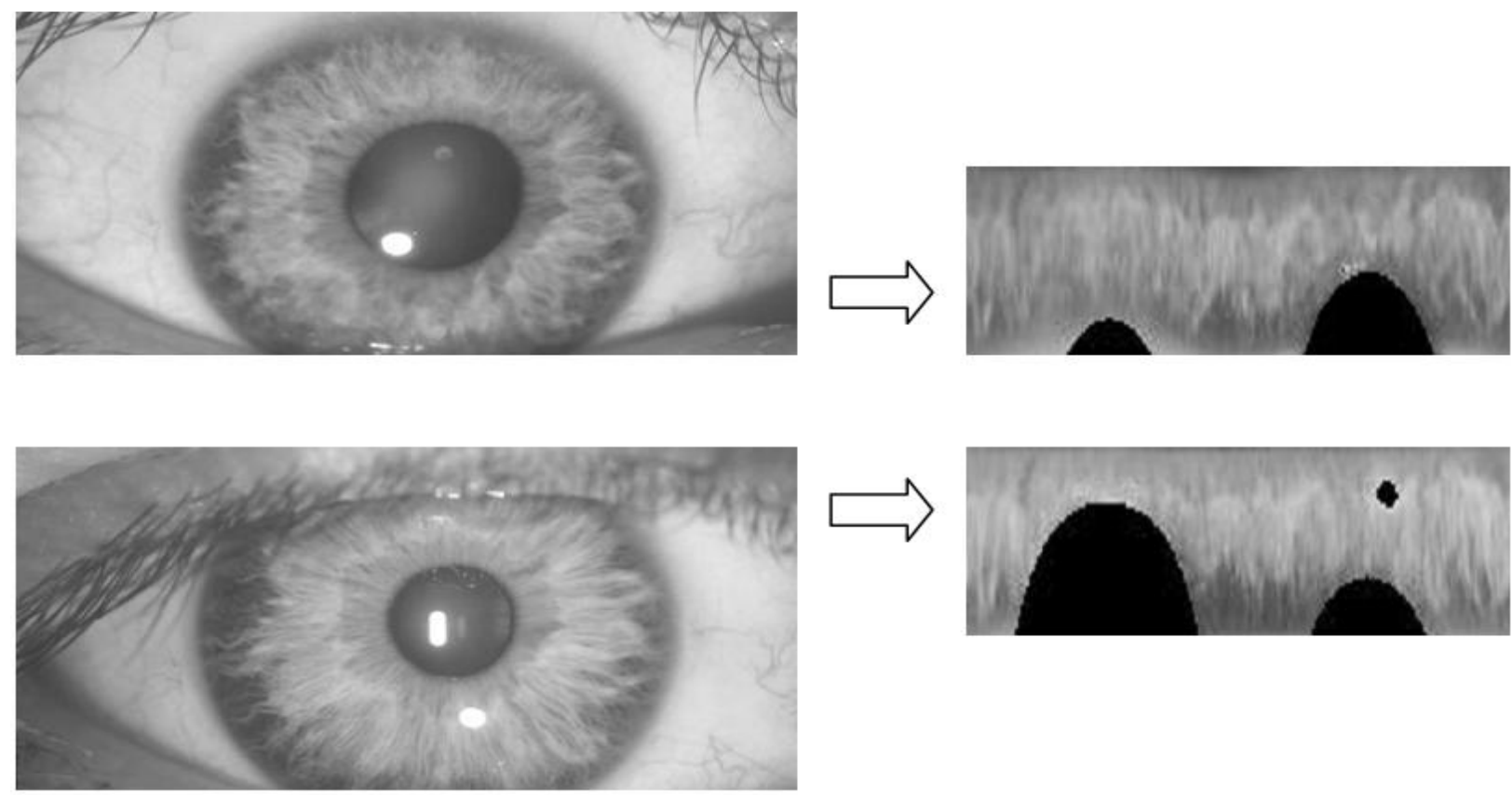

Figure 2.2: Illustration if the normalization process for two images of the same iris taken under varying conditions. Top image ,am 201be, bottom image, am201ge from the LEI database. Source: Daugman, (2003).

\subsection{Feature Encoding}

In order to provide accurate recognition of individuals, the most discriminating information present in an iris pattern must be extracted. Only the significant features of the iris must be encoded so that comparisons between templates can be made. Most iris recognition systems make use of a band pass decomposition of the iris image to create a biometric template.

\subsection{Matching}

The template that is generated in the feature encoding process will also need a corresponding matching metric, which gives a measure of similarity between two iris templates. This metric should give one range of values when comparing templates generated from the same eye, known as intra-class comparisons, and another range of values when comparing templates created from different irises, known as inter-class comparisons. These two cases should give distinct

\section{Volume 5 Issue 2, February 2016}




\section{International Journal of Science and Research (IJSR) \\ ISSN (Online): 2319-7064}

Index Copernicus Value (2013): 6.14 | Impact Factor (2014): 5.611

and separate values, so that a decision can be made with high confidence as to whether two templates are from the same iris, or from two different irises.

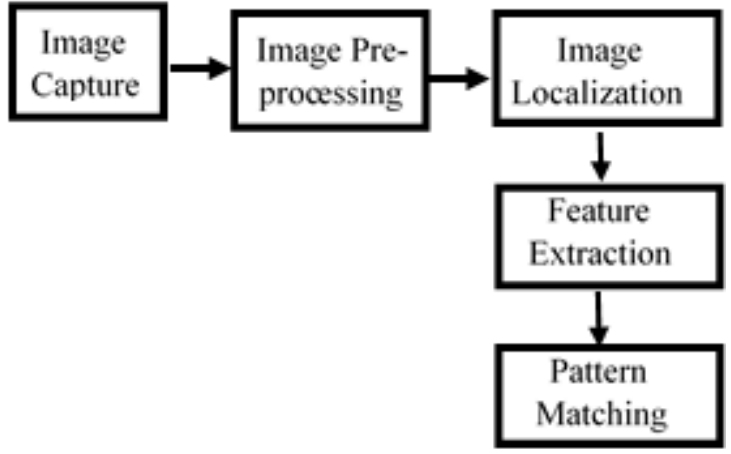

Figure 2.3: Block diagram of the stages of iris recognition system (Wolff E., 1976)

\subsection{Iris Segmentation}

The iris region, shown in figure 2.1 can be approximated by two circles, one for the iris/sclera boundary and another, interior to the first, for the iris/pupil boundary. The eyelids and eyelashes normally occlude the upper and lower parts of the iris region. Also, specular reflections can occur within the iris region corrupting the iris pattern. A technique is required to isolate and exclude these artifacts as well as locating the circular iris region.

\subsection{Adaptive Thresholding}

Thresholding is a technique used generally for detecting eyelashes, since they are dark compared to the surrounding regions. If the intensity value is less than the threshold value then the point belongs to the eyelashes. Eyelashes are of two types, separable eyelashes and multiple eyelashes that are grouped together. Separable eyelashes are detected using Gabor filters. If the resultant point is less than the threshold value then the point belongs to the eyelash. Multiple eyelashes are detected by calculating the variance of intensity. If the variance is less than the threshold, the center is taken as the point in the eyelash.

\subsection{Hough Transform}

The Hough transform is a standard computer vision algorithm that can be used to determine the parameters of simple geometric objects, such as lines and circles, present in an image. The circular Hough transform can be employed to deduce the radius and centre coordinates of the pupil and iris regions. An automatic segmentation algorithm based on the circular Hough transform was employed by Wildes et al, 1994, Kong and Zhang, 2001, Tisse et al., 2002. Firstly, an edge map is generated by calculating the first derivatives of intensity values in an eye image and then thresholding the result. From the edge map, votes are cast in Hough space for the parameters of circles passing through each edge point. A maximum point in the Hough space will correspond to the radius and center coordinates of the circle best defined by the edge points.

\subsection{Advantages of Iris over Other Biometric Features}

The iris of the eye has been described as the ideal part of the human body for biometric recognition has been one of the most effectively developed biometrics to ensure secure, efficient, and expedited airport operations for both landside and airside applications. Iris recognition's unique capabilities are proven to increase security, speed and user identification for several reasons;

- It is an internal organ that is well protected against damage and wear by a highly transparent and sensitive membrane (the cornea). This distinguishes it from fingerprints, which can be difficult to recognize after years of certain types of manual labour.

- The iris is mostly flat and its geometric configuration is only controlled by two complementary muscle (the sphinter pupillae and dilator pupillaer), which control the diameter of the pupil. This makes the iris shape far more predictable than, for instance that of face.

- The iris has a fine texture that - like finger - is determined randomly during embryonic gestation. Even genetically, identical individuals have completely independent iris textures, whereas DNA (generic "finger printing") is not unique for about $1.5 \%$ of the human population who has a genetically identical monozygotic twin.

- While there are some medical and surgical procedures that can affect the color and overall shape of the iris, the fine texture remains remarkable stable over many decades. Identifications have succeeded over a period of about 30 years.

\section{System Methodology and Design}

Circular Hough Transform is used to detect the presence of circular shapes in any image. For example detection of number of circular discs in an image. Another well-known application of Circular Hough Transform is the detection of number of coconuts in an image (Ritter N., 1999). Circular Hough Transform uses the parameterized equation of circle for this purpose. The equation can be written as

$$
(x-a)^{2}+(y-b)^{2}=r^{2}
$$

Where $\mathrm{x}, \mathrm{y}$ are the points on the circumference of the circle, $\mathrm{a}, \mathrm{b}$ is the centre of circle and $\mathrm{r}$ is the radius of the circle.

The equation of circle can be written as

$$
\begin{gathered}
x=a+r * \cos (\theta) \\
y=b+r * \sin (\theta)
\end{gathered}
$$

Circular Hough Transform uses these equations to compute the $\mathrm{CHT}$ of a circle and detect the presence.

The algorithm for CHT is follows

Read an image file.

Find edges in the image.

Define a range of radius to be used.

For each edge point, Draw a circle with that edge point as the center and a radius $r$ and increment the number of votes by 1 for all the coordinates that coincide with the circumference of the circle drawn, in the accumulator 


\section{International Journal of Science and Research (IJSR) \\ ISSN (Online): 2319-7064}

Index Copernicus Value (2013): 6.14 $\mid$ Impact Factor (2014): 5.611

space. Find circles for an edge point for all the radius in the range.

Find the maximum number of in the accumulator space.

Plot the circle with parameters $(\mathrm{r}, \mathrm{a}, \mathrm{b})$ corresponding to the maximum in the accumulator space.

The circle obtained is the desired circle with $(r, a, b)$ as the radius and center of circle respectively.

\subsection{Image Acquisition}

The first phase of the work was to collect a large database consisting of several iris images from various individuals. CASIA Iris Image Database was used. CASIA Iris Image Database (CASIA-Iris) was developed by Chinese Academy of Sciences Institute of Automation research group. Among the public and freely available iris image database for biometric purpose is the CASIA iris database, the most widely used for iris biometric purpose (captured under good positioning and illumination condition). However, its images incorporate few types of noise, almost exclusively related with eyelid and eyelash obstruction. CASIA images were deliberately taken for biometric issues and standardized for such. But, in normal life biometric activities where user"s non-cooperation is expected, heterogeneity of image is mostly obtained.

\subsection{The User Interface}

The user interface (front end) was designed using MATLAB. It conjugates all separate tasks involved in the evaluation process on a single user friendly interface allowing a user to perform the various stages in sequential ways.

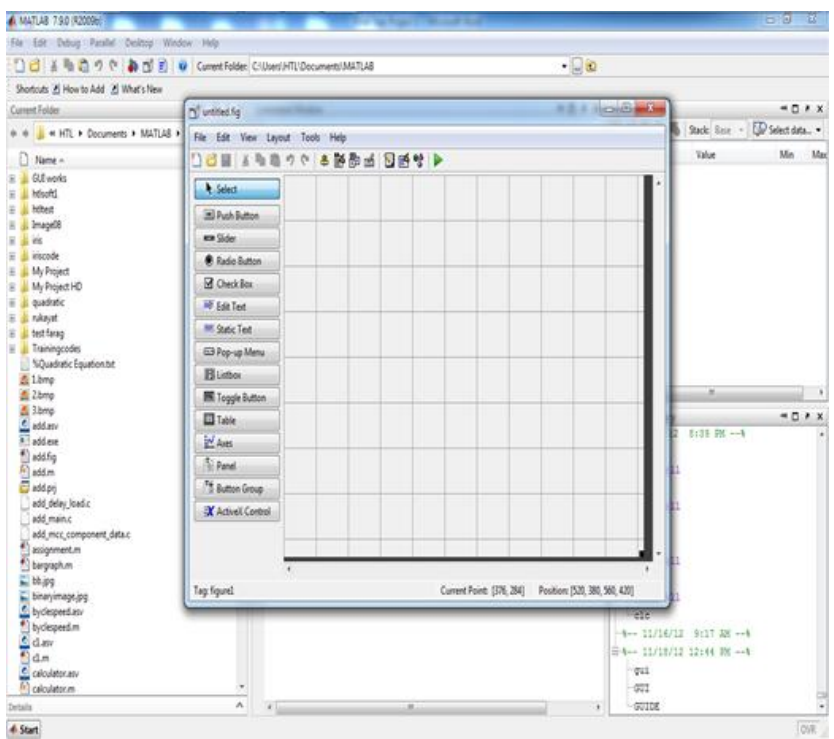

Figure 3.1: The GUI in MATLAB

The interface uses its input from a database of captured iris images which serves as the registered or authorized individual of the security concerns.

\subsection{System Design}

The design interface was implemented with. The designs as shown below gives stepwise method of the way the system implements the segmentation and the recognition processes. This helps to secure the system use.

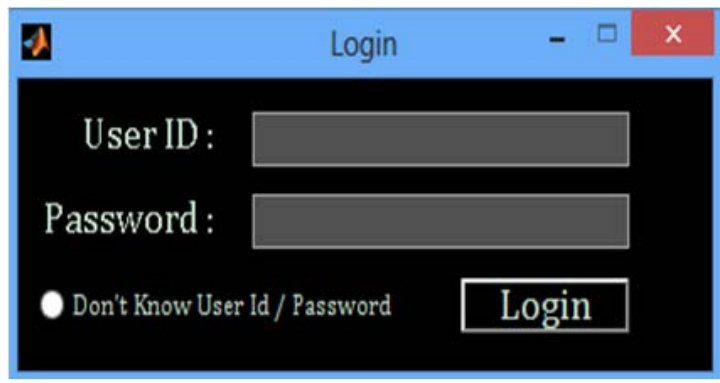

Figure 3.2: The system login interface

The implementation of this work was done by the design of the interface shown below.

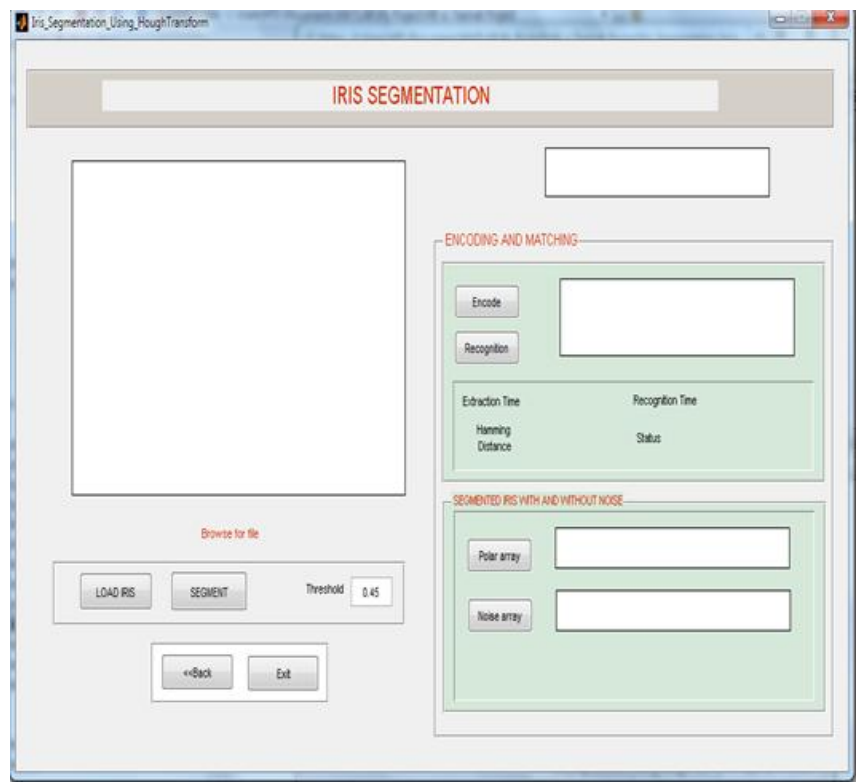

Figure 3.3: The user interface at the first stage of execution

The first step of the algorithm is to input an iris image, specifically CASIA Iris images. This iris images were stored in the database which the system can easily browse and locate for the recognition. In MATLAB, a reading task behind the screen was used with the command imread('image', file extension), and imshow(,image ", file extension) to show the iris image on the tab meant for it on the interface. The figure below shows the interface for the iris image read into the system for recognition. 


\section{International Journal of Science and Research (IJSR) \\ ISSN (Online): 2319-7064}

Index Copernicus Value (2013): 6.14 | Impact Factor (2014): 5.611

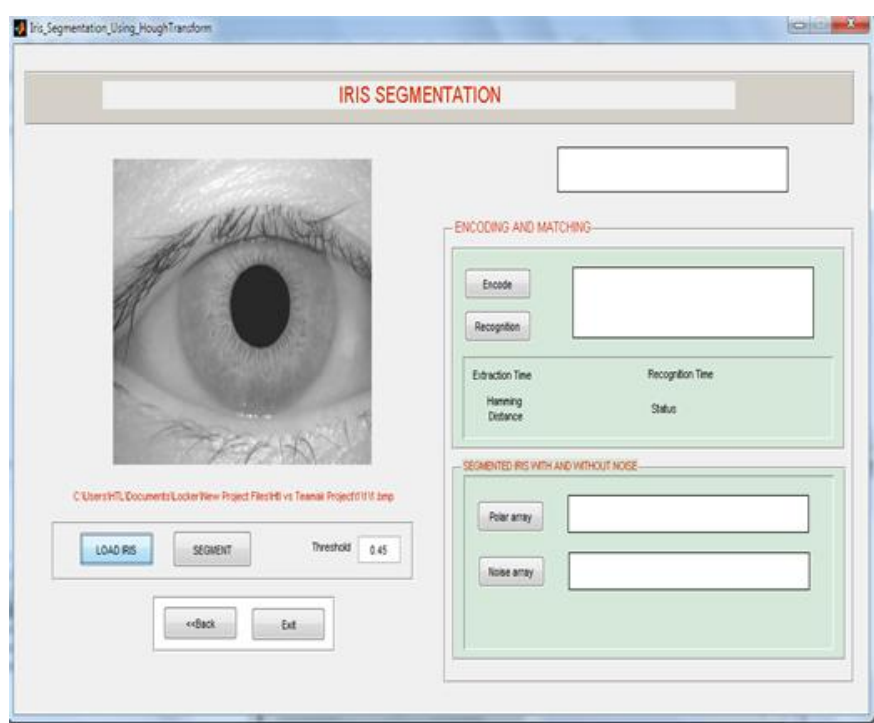

Figure 3.4: Iris Image read into the system for Recognition

Now, before any further step can be taken, the iris image has to be converted to gray scale. The initial format of the original image is in RGB, and the iris image is set for the image pre-processing. MATLAB can only process a 2-D dimensional image and so it is necessary that the iris image is converted for further image pre-processing. This leads to the iris normalization which enhances the iris image for further stages in recognition process. However, to make the interface tidy and compact, the codes for image conversion to gray scale is located into the segmentation command.

The last stage is the cross-referencing the template with the database iris images. At stage of the recognition, the matching of the iris images is carried out. The matching extracted feature of the iris image is displayed. The figure below shows the last stage of the recognition process after the image has been recognized.

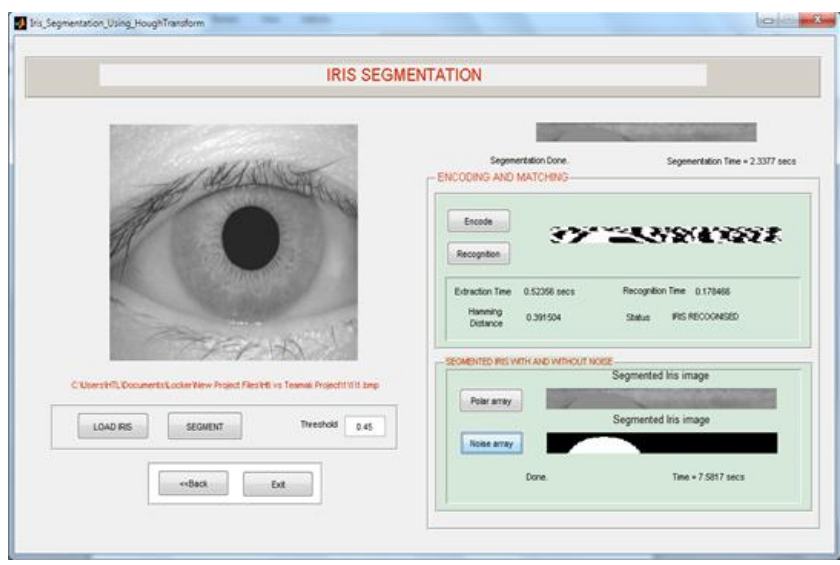

Figure 3.5: The interface of a recognized iris image and process

This stage marks the third evaluation area. The recognition time is examined at the end of this stage via the "Recognition Time" column of the pin interface.

\subsection{The Segmentation Process}

To detect the iris and pupil boundaries accurately, Circular Hough transform is applied first to the iris-sclera boundary and the to the iris-pupil boundary, since pupil lies within the iris region, it involves canny edge detection for generating an edge map.
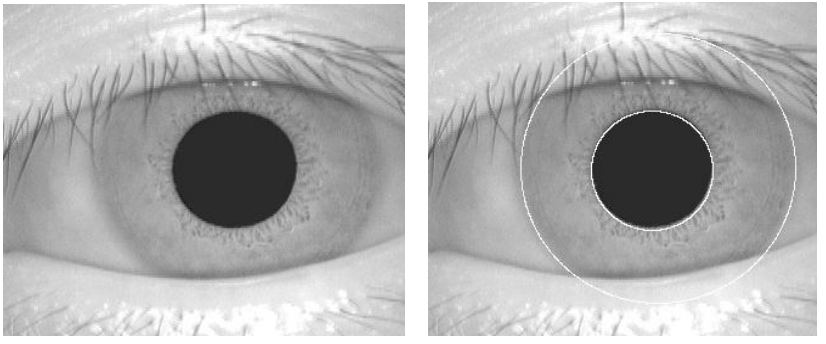

Figure 3.6: Showing iris image and circular Hough transform segmented image

In performing the edge detection, gradients are biased in the vertical direction for the iris-sclera boundary, and in horizontal and vertical direction for the iris-pupil boundary. From the gradient image, weak edges are eliminated by thresholding. If the intensity values are greater than the high threshold then those pixels are considered as edges and if the intensity values are less than the lower threshold, then those pixels are considered as weak edges and eliminated. If the intensity values lie the high and low threshold, then the average of its neighboring pixels is taken and if the value is greater than the lower threshold, then the pixel is considered as the edge point. After eliminating weak edges, radius and center coordinates for iris and pupil are calculated from the edge map by applying Circular Hough transform.

Eyelids were isolated drawing a line to the upper and lower eyelids using the linear Hough transform. A second horizontal line is then drawn intersecting the first line at the edge of the iris that is closest to the pupil, to isolate maximum of eyelid regions. These lines lie exterior to the pupil and interior to the iris. This process is shown in Figure 3.6 and is done for both the eyelids (top and bottom.) canny edge detection is used for generating and edge map and only horizontal gradient information is taken for detecting the eyelids. Linear Hough transform was used to detect the upper and lower eyelids. If the maximum value in the Hough space is less than the threshold, then no lines are drawn.
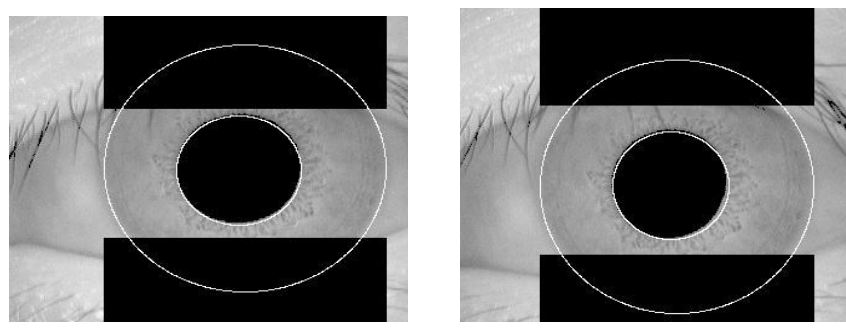

Figure 3.9: segmentation using Linear Hough transform

For isolating eyelashes, a simple thresholding technique was used, since eyelashes are dark when compared with the rest of the eye image. The segmented iris region is normalized and unwrapped into a rectangular block with fixed dimensions, and encoded, extracted the matched. The system flowchart is shown below 


\section{International Journal of Science and Research (IJSR)}

ISSN (Online): 2319-7064

Index Copernicus Value (2013): 6.14 | Impact Factor (2014): 5.611

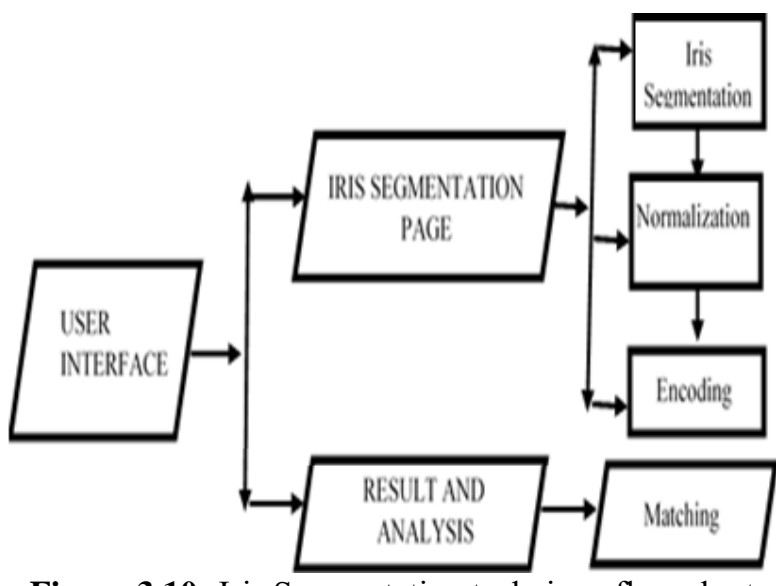

Figure 3.10: Iris Segmentation technique flow chart

\section{Results and Discussion}

Iris segmentation is a key in an iris recognition system. And iris segmentation result determines the efficiency and accuracy. Segmentation is the most critical stage of iris recognition system because data that is wrongly represented as iris pattern will corrupt the generated biometric template.

The system was implemented using MATLAB 7.0 in windows 7 operating system environment on Intel Pentium processor, $2.13 \mathrm{GHZ}$ and 1GB RAM. In this approach 120 gray scale iris images of 20 individual from the Chinese Academy of Science Institute of Automation (CASIA) was used. Evaluations were made at two different areas or point in the course of the system execution. The first was at a point of segmentation where the segmentation time of the technique under examination was observed and recorded. The second area of evaluation takes place at the point of template matching meant for observing and recording the recognition time.

The Evaluation or tests were made on various iris images available in the database, ensuring that each of them was examined using the hybrid segmentation technique. The segmentation results of 15 iris images from 15 different individual tests are presented in the Table below. Where $0=$ Not Recognized and $1=$ Recognized

Table4.1: Segmentation results of 15 iris images from different individuals

\begin{tabular}{|c|c|c|c|c|}
\hline $\begin{array}{c}\text { Iris } \\
\text { tested }\end{array}$ & $\begin{array}{c}\text { Hamming } \\
\text { Distance }\end{array}$ & $\begin{array}{c}\text { Segmentation } \\
\text { Time (secs) }\end{array}$ & $\begin{array}{c}\text { Recognition } \\
\text { Time (secs) }\end{array}$ & Status \\
\hline 1 & 0.3915 & 2.2634 & 0.0324 & 1 \\
\hline 2 & 0.4247 & 1.8342 & 0.0944 & 1 \\
\hline 3 & 0.3707 & 2.4095 & 0.0645 & 1 \\
\hline 4 & 0.3539 & 1.6794 & 0.0742 & 1 \\
\hline 5 & 0.3588 & 2.2039 & 0.0316 & 1 \\
\hline 6 & 0.3878 & 1.5965 & 0.0731 & 1 \\
\hline 7 & 0.3505 & 2.0054 & 0.0462 & 1 \\
\hline 8 & 0.355 & 1.9656 & 0.0304 & 1 \\
\hline 9 & 0.4069 & 2.4376 & 0.0303 & 1 \\
\hline 10 & 0.3884 & 1.7121 & 0.0298 & 1 \\
\hline 11 & 0.3908 & 3.1919 & 0.0308 & 1 \\
\hline 12 & 0.3805 & 1.9699 & 0.0321 & 1 \\
\hline 13 & 0.3686 & 2.7049 & 0.0304 & 1 \\
\hline 14 & 0.3927 & 2.2675 & 0.0314 & 1 \\
\hline
\end{tabular}

\begin{tabular}{|c|c|c|c|c|}
15 & 0.3623 & 2.6286 & 0.0325 & 1 \\
\hline 16 & 0.4462 & 1.8169 & 0.0334 & 1 \\
\hline 17 & 0.4108 & 2.1361 & 0.0315 & 1 \\
\hline 18 & 0.3689 & 1.6741 & 0.0305 & 1 \\
\hline 19 & 0.37209 & 2.0057 & 0.0305 & 1 \\
\hline 20 & 0.374 & 1.7328 & 0.0324 & 1 \\
\hline
\end{tabular}

The table below shows the average segmentation time and average recognition time.

Table 4.2: Average segmentation and recognition time

\begin{tabular}{|c|c|}
\hline Total Iris tested & 20 \\
\hline Average Segmentation Time & $0.21089 \mathrm{Secs}$ \\
\hline Average Recognition Time & $0.04113 \mathrm{Secs}$ \\
\hline
\end{tabular}

\section{\begin{tabular}{|l|l|}
\hline Average Recognition Time & 0.04113 Secs \\
\hline
\end{tabular}}

The average Segmentation time was calculated thus:

Average Segmentation time $=\frac{\text { Total sum of segmentation time }}{\text { Numbers of segmentation time }}$

The average Recognition time was calculated thus:

Average Recognition Time $=\frac{\text { Total sum of recognition time }}{\text { Numbers of recognition time }}$

Table 4.1 and 4.2 clearly shows that the developed hybrid segmentation technique for iris recognition using Circular Hough and Linear Hough transform algorithm has a faster segmentation and recognition time. From the status column on the table shows that the entire 20 iris segmented was recognized when matched which is not bad for individual to go for iris authentication.

For CASIA database with 20 individuals, six (6) iris images were used per person, three (3) on the left eye and three (3) on the right, total of 120 iris image. False Acceptance Rate (FAR) means probability of identifying an imposter as an enrolled user. This means that, for every interclass match considered with CASIA, a total of $119 \times 6$ comparisons were done on each eye sample, a common threshold value obtained from analysis of each iris image was also used to evaluate the performance of the algorithm. Matching time describes only the average time taken by the program run in MATLAB to compare the digital codes of the iris images, done at matching stage alone while Average Recognition Time measures the average total time taken to segment, normalize, extract, and encode the features of a chosen iris and match with a chosen set of templates in a range. For intra-class recognition however, a chosen iris in a class is compared only with other samples in its class from the first subject to the last and since this image are from the same set of people, the likely error here would be due to a False Reject Rate (FRR). This investigation was repeated using different hamming distance in order to arrive at optimum settings for the recognition system.

Figure 4.1a below show the plotting of Average Segmentation and Recognition time i.e. plotting of segmentation time against number of tested iris for Average Segmentation Time and figure $4.1 \mathrm{~b}$ shows the plotting of recognition time against number of tested iris for Average 


\section{International Journal of Science and Research (IJSR) \\ ISSN (Online): 2319-7064}

Index Copernicus Value (2013): 6.14 | Impact Factor (2014): 5.611

\section{Recognition Time.}

Figure 4.1a: shows Average Segmentation Time, Figure 4.1b: shows Average Recognition Time

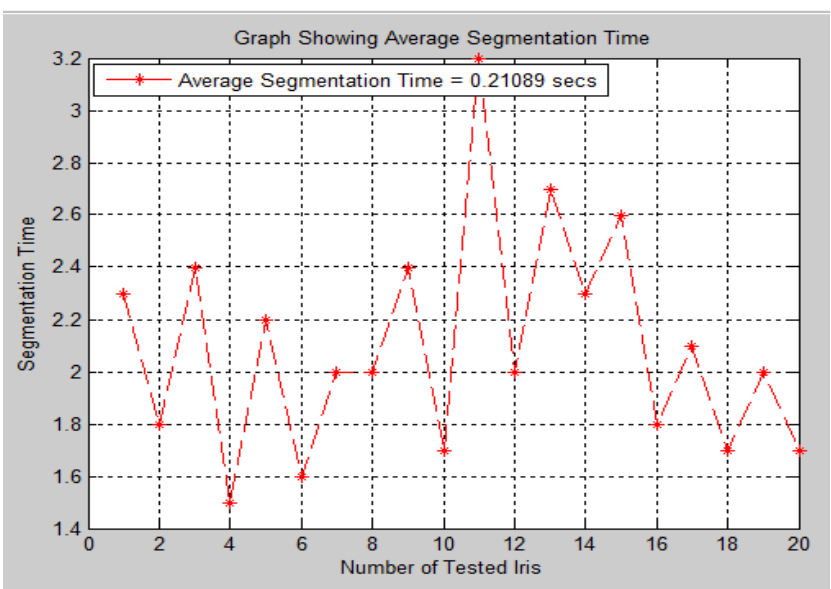

Figure 4.1a

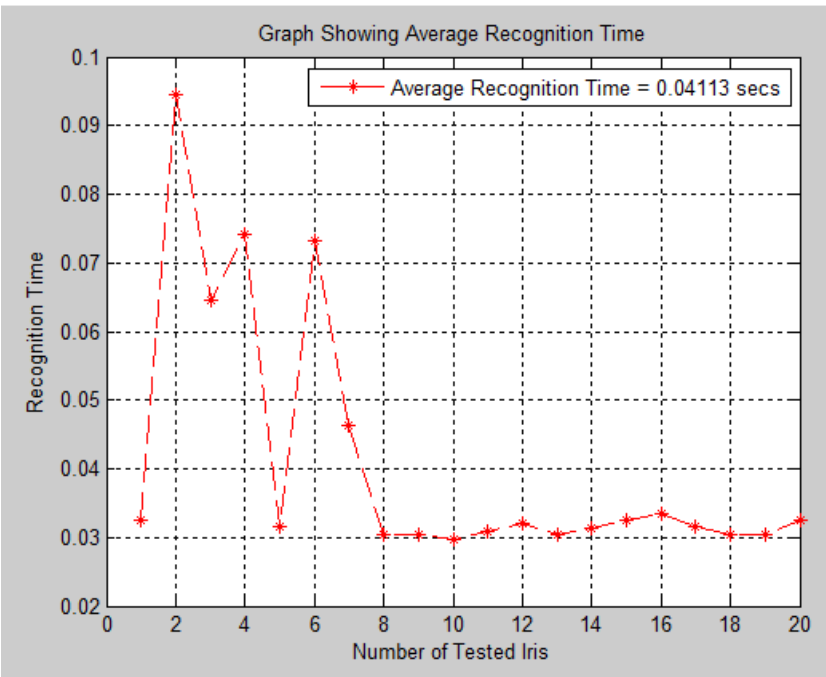

Figure 4.1b

The figure $4.2 \mathrm{a}$ below shows the graph of the interclass matching i.e. plotting the hamming distance against false acceptance rate (FAR), and figure $4.2 \mathrm{~b}$ shows the graph of the Interclass matching i.e. plotting hamming distance against false rejection rate (FRR).

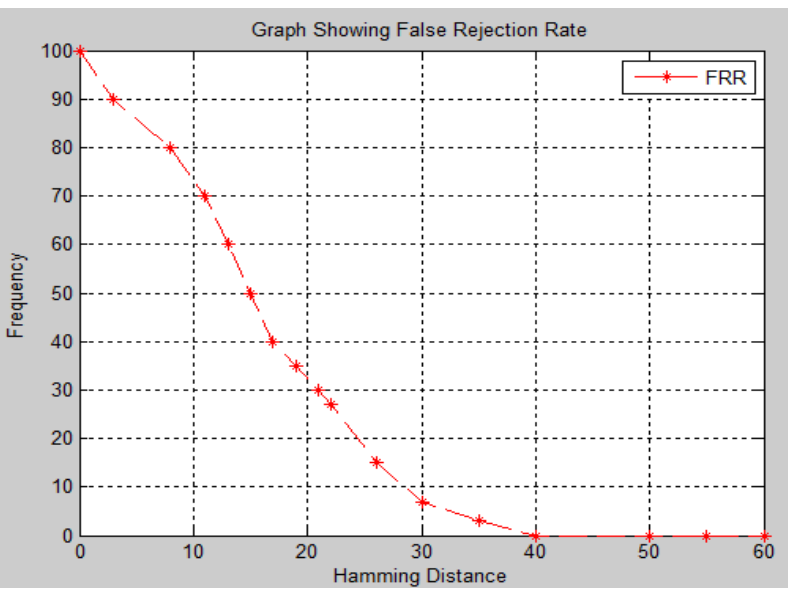

Figure 4.2a: plot of hamming distance against false acceptance rate (FAR)

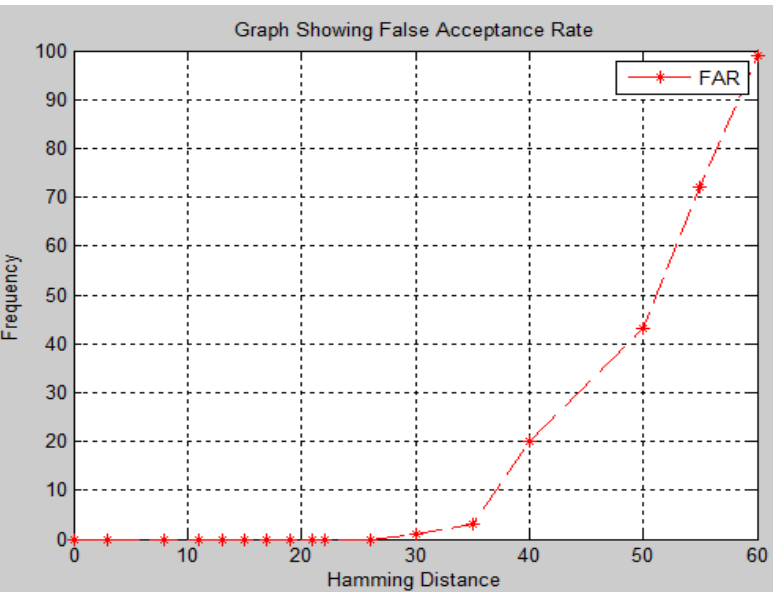

Figure 4.2b: plot of hamming distance against false rejection rate (FRR).

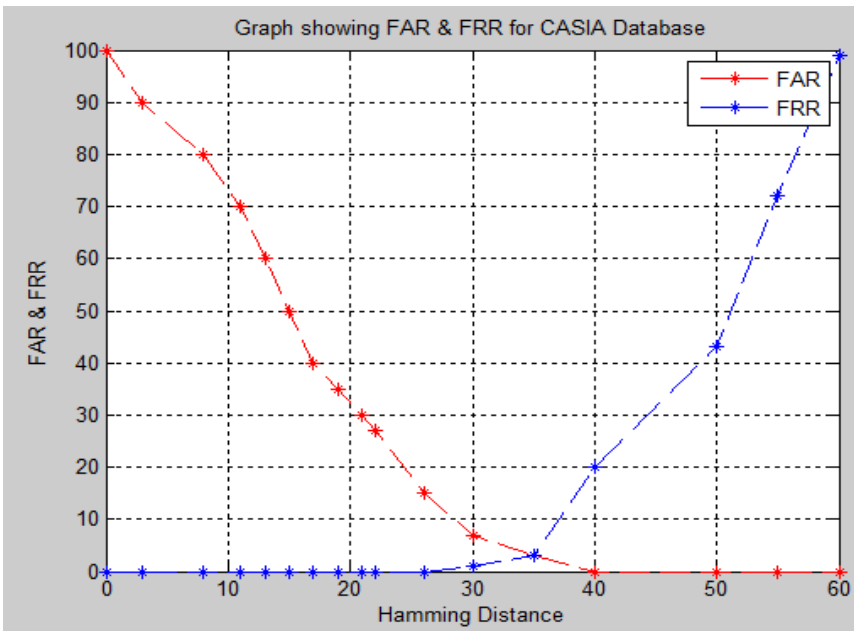

Figure 4.3: Graph showing FAR \& FRR for CASIA Database

Figure 4.3 showed a combined graph of FAR and FRR. The area of intersection between the two distributions represents errors in the system performance.

\section{Other Recommendations}

\subsection{Conclusion}

In this work, new hybrid system was developed using CASIA irises (the most widely used). The system performance was evaluated in terms of the segmentation time, recognition time, False Acceptance Ratio (FAR), False Rejection Rate (FRR), Interclass and Intra-class matching scores. The average segmentation time is 0.21089 seconds and the average recognition time is 0.04113 seconds which is not bad for an authentication system. It was observed that segmentation is the most critical stage of iris recognition system because data that is wrongly represented as iris pattern will corrupt the generated biometric template with the CASIA database of 20 individual, $99 \%$ of the images are segmented properly. The method gave a better recognition rate of $99.9 \%$ with false acceptance and rejection ratio of 0.00877 and 0.100 respectively and also consumes less time for the matching of template. 


\section{International Journal of Science and Research (IJSR) \\ ISSN (Online): 2319-7064}

Index Copernicus Value (2013): 6.14 | Impact Factor (2014): 5.611

\subsection{Recommendation}

In the course of this work, we discovered that the iris segmentation is the most difficult stage out of all the stage involved in the iris recognition system. Other upcoming researchers should explore more on the possibilities of using other segmentation. For real implementation of the iris Recognition system which would be embedded in security, it is hereby recommended that iris camera with LED light should be used. The system did not cater for acquisition of iris image of a new user that did not have his/her iris image saved on the database. Therefore, provision should be made for iris image capturing and saving of new user for authentication.

\section{References}

[1] Adegoke, B. O., Omidiora, E. O. Falohun, S. A. and Ojo, J.A. (2013). Iris Segmentation: a survey. International Journal of Modern Engineering Research (IJMER) Vol.3, Issue.4, Jul - Aug. 2013 pp-1885-1889 ISSN: 2249-6645

[2] Boles, W. and Boashash , B. (1998), "A Human Identification Technique using Images of the iris and wavelet Transform", IEEE Transaction on Signal Processing, 46 (4): 457-459.

[3] Daugman J., (1993). High confidence visual recognition of person "e by a test of statistical independence.IEEE Transaction, PAMI, 15(11), pp. 1148-1161

[4] Daugman J. (2002) "How iris recognition works".Proceedings of 2002 International Conference on Image Processing, Vol 1.

[5] Daugman J. (2003). The importance of being random: Statistical principles of the Iris Recognition. Pattern Recognition 36(2): 279-29.

[6] Kong W. and Zhang D., (2001). Accurate iris segmentation based on novel reflection and eyelash detection model. Proceedings of 2001 International Symposium on Intelligent Multimedia, Video and Speed Proceeding, Hong Kong.

[7] Lim S., Lee K., Byeon O. and Kim J., (2001). Efficient iris recognition through improvement of feature vector and classifier. ETRI Journal, 23(2), pp. 61-70.

[8] Ma, L., T. Tan, Y. Wang and D. Zhang, (2004). Efficient iris recognition by characterizing key local variations. IEEE Trans., Image Processing, 13: 739-750.

[9] Nakissa B. and Mohammad S.M., (2008). A new approach for iris localization in iris recognition systems. IEEE Transaction, 5(8), pp. 516-523.

[10] Ritter N. (1999). Location of the pupil-iris border in slitlamp images of the cornea. Proceedings of the International Conference on Image Analysis and Processing.

[11] Tisse C., Martin L., Toress L., and Robert M., (2002) "Personal Identification technique using human iris recognition", In proceedings of ICVI ${ }^{\text {ee }} 02$, pp.294 -299.

[12] Peihua L. and Xiaomin L., (2008). An incremental method for accurate iris segmentation. Proceedings of IEEE

[13] Prabhakar A., Sanderson S., Erbetta J., (2000). Authentication for secure environments based on iris scanning technology. IEEE Colloquium on Visual Biometrics.

[14]Wildes R. (1997) "Iris Recognition: An Emerging Biometric Technology", proceeding of the IEEE, 85(a): $1348-1363$.

[15] Wolff E., (1976), Anatomy of the eye and orbit, $7^{\text {th }}$ edition. H. K. Lewis \& Co. Ltd, New York.

\section{Author Profile}

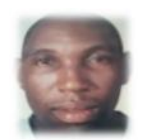

Olusegun O. Lawal received his B.Sc. degree in Mathematical Sciences (Computer Science option) from Federal University of Agriculture Abeokuta in 1998 and M.Sc. degree in Computer Science from University of Ibadan in the year 2010 . He is currently a $\mathrm{PhD}$ student at Ladoke Akintola University, Ogbomosho where he is carrying out a research on ontology based knowledge discovery for traditional herbs. He is a lecturer in the Department of Computer Science, Joseph Ayo Babalola University, Nigeria. His research interest includes Information modeling, e-health, e-learning and System Security.

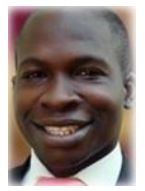

Babajide O. Afeni received his B.Sc. degree in Computer Science from Joseph Ayo Babalola University, Ikeji - Arakeji, Nigeria in 2011 and M. Tech. degree in Computer Science from The Federal University of Technology, Akure, Nigeria in the year 2015. He is currently a PhD student at The Federal University of Technology, Akure and a lecturer in the Department of Computer Science, Joseph Ayo University, Ikeji - Arakeji. His research interest includes Machine Learning, System Security, Elearning and Geographic Information System (GIS).

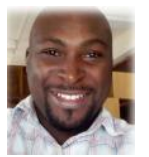

Moshood O. Lawal received his B.Sc. degree in Computer Science from Joseph Ayo Babalola University, Ikeji - Arakeji, Nigeria in 2015. He currently works with The Federal Polytechnic, Ede, Nigeria. His research interest includes Information and System Security. 